\title{
ANÁLISE DO PERFIL EPIDEMIOLÓGICO DOS ÓBITOS NO ESTADO DO PIAUÍ ENTRE 2010- 2015
}

\author{
ANALYSIS OF THE EPIDEMIOLOGICAL PROFILE OF DEATHS IN THE STATE OF PIAUÍ BETWEEN 2010-2015 \\ Eduardo Andrade Vasconcelos ${ }^{1}$, Augusto César Evelin Rodrigues ${ }^{2}$ \\ ${ }^{1}$ Faculdade FACID/WYDEN, discente do curso de medicina, Trabalho de Conclusão de Curso (TCC), ano de defesa: 2019. \\ Teresina, Piauí, Brasil. Email: eduandradev.96@gmail.com. \\ ${ }^{2}$ Augusto César Evelin Rodrigues, docente do curso de Medicina da Faculdade FACID/WYDEN, Teresina, Piauí, Brasil. Email: \\ augustocevelin@yahoo.com.br.
}

\begin{abstract}
OBJECTIVE: To analyze the deaths in the state of Piauí between 2010-2015, to measure infant mortality, to identify deaths according to sex and age and to classify according to causes. METHODS: documentary, retrospective and quantitative research, using data recorded in SIM, from the public database of the computer department of SUS. RESULTS: there was a progressive increase of 3.5\% in the number of deaths reported in Piaui during this period. Most child deaths were among children under one year, especially in newborns. It was found that men were the most affected in all age groups. Among the causes of death, firstly circulatory system diseases, then neoplasms and third external causes. CONCLUSION: The state of Piauí follows the worldwide trend of epidemiological and demographic transition, so public policies aimed at preventing deaths should follow this change. Although infant mortality has decreased prenatal, childbirth and newborn care is still needed.
\end{abstract}

Keywords: Mortality Registries. Infant Mortality. Cause of Death. Health Transition.

\section{RESUMO}

OBJETIVO: analisar os óbitos no estado do Piauí entre 2010-2015, mensurar a mortalidade infantil, identificar os óbitos segundo sexo e faixa etária e classificar segundo as causas. MÉTODOS: pesquisa documental, retrospectiva e quantitativa, usou-se dados registrados no SIM, oriundos do banco de dados público do departamento de informática do SUS. RESULTADOS: houve aumento progressivo de 3,5\% no número de óbitos notificados no Piauí nesse período. A maioria dos óbitos infantis esteve entre crianças menores de um ano, principalmente nos neonatos. Identificou-se que os homens foram os mais afetados em todas as faixas etárias. Entre as causas da morte, em primeiro lugar as doenças do sistema circulatório, depois neoplasias e terceiro causas externas. CONCLUSÃO: o estado do Piauí segue a tendência nacional e mundial de transição epidemiológica e demográfica, portanto, as políticas públicas voltadas para prevenção de mortes devem seguir essa mudança. Apesar da mortalidade infantil ter diminuído, ainda é necessário melhorar a assistência pré-natal, ao parto e ao recém-nascido.

DESCRITORES: Registros de Mortalidade. Mortalidade Infantil. Causas de Óbito. Transição epidemiológica. 


\section{Como citar este artigo:}

Vasconcelos EA, Rodrigues ACE. Análise do perfil epidemiológico dos óbitos no estado do Piauí entre 20102015. J. Ciênc. Saúde [internet]. 2018 [acesso em: dia mês abreviado ano];1(3):59-70. Disponível em: DOI: https://doi.org/10.26694/2595-0290.20181359-709662 
INTRODUÇÃO

As doenças crônicas não transmissíveis (DCNT) constituem o maior problema global de saúde e têm gerado um elevado número de mortes prematuras, perda de qualidade de vida com alto grau de limitação e incapacidade, além de serem responsáveis por impactos econômicos para famílias, comunidades e sociedade em geral $^{(1)}$.

O Brasil participou ativamente da mobilização global contra as DCNT ao lançar o "Plano de Ações Estratégicas para o Enfrentamento das Doenças Crônicas Não Transmissíveis no Brasil, 2011-2022", o qual define metas e compromissos, ações e investimentos no sentido de preparar o país para o enfrentamento dos desafios representados por aquelas doenças e seus fatores de risco nos próximos 10 anos. Dentre as metas traçadas, tem-se a redução de $2 \%$ ao ano na mortalidade devido às quatro principais causas de mortalidade por DCNT ${ }^{(2)}$.

Outra causa de mortalidade é a violência, fenômeno que sempre fez parte da experiência humana. Estima-se que, a cada ano, as causas violentas sejam responsáveis por mais de um milhão de mortes no mundo e, principalmente, entre 15 e 44 anos de idade em homens ${ }^{(3)}$.

A causa de violência e letalidade no Brasil é multifatorial. Fatores como a desigualdade socioeconômica, déficit na segurança pública, criminalidade, porte de arma de fogo são referidos diariamente nas taxas de mortalidade, principalmente nas regiões Nordeste e Norte do Brasil, visto que são problemas que desafiam as instituições governamentais e a convivência em sociedade ${ }^{(4)}$.

No Brasil, o monitoramento da violência para fins de vigilância epidemiológica é realizado por meio da análise dos dados da declaração de óbito (DO) e da autorização de internação em hospitais públicos - ou autorização de internação hospitalar (AIH), fornecidos, respectivamente, pelo Sistema de Informações sobre
Mortalidade (SIM) e Sistema de Informações Hospitalares do Sistema Único de Saúde (SIH/SUS). Ambos os registros são gerenciados pelo Ministério da Saúde (MS) e constituem importante ferramenta para o conhecimento da carga da violência no país ${ }^{(5)}$.

Além disso, o óbito infantil é um importante indicador da saúde de mulheres e crianças, constituindo-se em um evento sentinela devido à evitabilidade. Relacionase aos determinantes biossocioculturais e assistenciais e a redução depende da melhoria efetiva nas condições de vida e de políticas públicas de saúde. As falhas na atenção à gestante, ao parto e ao recém-nascido são particularmente relevantes na ocorrência dos óbitos no primeiro ano de vida ${ }^{(6)}$.

A taxa de incidência global de câncer mostrado pelo WHO através do relatório GLOBOCAN foi de 18.1 milhões de pessoas. A taxa de mortalidade foi de 9.6 milhões de casos, destes, 1.371 .000 (14.4\%) nas Américas, sendo este o continente mais acometido ${ }^{(1)}$.

Em relação à mortalidade devido ao câncer, considerando os óbitos segundo o gênero, de acordo com o WHO, a maior taxa de incidência global de câncer nas mulheres deu-se na mama (24,2\%), seguido do colorretal $(9,4 \%)$. Em relação à taxa de mortalidade global no gênero feminino, em primeiro lugar foi mama (15\%) seguido do pulmão (13,8\%). Já no sexo masculino, a maior incidência foi no pulmão $(14,5 \%)$ seguido da neoplasia de próstata (13,5\%). Em relação à mortalidade neste sexo, câncer no pulmão correspondeu a $22 \%$ dos casos seguido do fígado $(10,2 \%)^{(1)}$.

O problema gerador da questão de pesquisa foi o fato de que medir quantas pessoas morrem a cada ano e por que elas morreram é um dos meios mais importantes juntamente com a avaliação de como as doenças e lesões estão afetando as pessoas - para avaliar a eficácia do sistema de saúde de um país, fato este tão estudado pela OMS.

O conhecimento sobre a realidade do perfil epidemiológico da mortalidade no estado do Piauí é importante para auxiliar as autoridades das instituições 
de saúde na elaboração de políticas públicas no intuito de prevenir o maior número possível de óbitos através da conscientização da população piauiense, tornandose, assim, evidente a relevância do presente estudo.

Dessa forma, o objetivo geral deste estudo foi analisar os óbitos ocorridos no estado do Piauí no período de 2010 a 2015. Os objetivos específicos foram mensurar a mortalidade infantil no Piauí, identificar os óbitos segundo sexo e faixa etária e classificar os óbitos segundo as causas.

\section{METODOLOGIA}

A pesquisa foi desenvolvida de acordo com a Resolução 466/2012 do Conselho Nacional de Saúde, que define as diretrizes e normas regulamentadoras de pesquisas, envolvendo seres humanos.

Devido ao fato de que a base de dados que foi utilizada ser de domínio público, inexiste a necessidade do envio do projeto de pesquisa ao CEP-FACID (Comitê de Ética em Pesquisa da Instituição

Tratou-se de uma pesquisa epidemiológica do tipo documental, retrospectiva e quantitativa sobre o perfil epidemiológico dos óbitos no estado do Piauí, compreendido entre os anos de 2010 a 2015.

Foram incluídos todos os pacientes que vieram a óbito no estado do PI no período de 2010 a 2015. Foram excluídos as notificações que foram realizadas no estado do Piauí, mas que eram de pacientes que residiam em outro estado.

O universo da pesquisa compreendeu um total de 106.031 notificações, registradas no Sistema de Informações sobre Mortalidade (SIM), de óbito do estado do Piauí, compreendido entre o período de 2010 a 2015. As variáveis que foram analisadas são: faixa etária, sexo e causas.
Os dados foram coletados no período de Agosto de 2018. A pesquisa foi realizada com base nos dados registrados no SIM (Sistema de Informações sobre Mortalidade), oriundos da banco de dados público do departamento de informática do SUS - DATASUS, onde foram pesquisados os critérios pré-estabelecidos. Em seguida, o banco de dados foi registrado no software Microsoft Excel ${ }^{\circledR} 2013$ Redmond, WA, EUA.

Após a coleta, os dados foram analisados através de estatística básica, de frequências absoluta e relativa e cálculos de incidência, sendo distribuídos conforme os objetivos estabelecidos. Finalmente, foram apresentados em forma de tabelas e gráficos e discutidos à luz da literatura.

O estudo não trouxe nenhum risco para os participantes, uma vez que se tratou de dados de domínio público. O único risco possível seria a identificação dos pacientes que foram a óbito, no entanto isso não ocorreu porque são dados que não possuem a denominação do paciente.

A pesquisa trouxe como benefício permitir entender o perfil epidemiológico dos óbitos no estado do Piauí no período citado, auxiliando, assim, no entendimento do processo que envolve a mortalidade nesse Estado.

\section{RESULTADOS}

Quadro 1 - Óbitos por ano, Piauí, 2010-2015

\begin{tabular}{|c|c|c|}
\hline $\begin{array}{c}\text { Ano do } \\
\text { Óbito }\end{array}$ & $\begin{array}{c}\text { Óbitos por } \\
\text { Residência }\end{array}$ & $\%$ \\
\hline 2010 & 15614 & $14,7 \%$ \\
\hline 2011 & 17052 & $16,1 \%$ \\
\hline 2012 & 17449 & $16,5 \%$ \\
\hline 2013 & 17985 & $17,1 \%$ \\
\hline 2014 & 18565 & $17,5 \%$ \\
\hline 2015 & 19366 & $18,3 \%$ \\
\hline Total & $106031(\mathrm{n})$ & $100 \%$ \\
\hline
\end{tabular}

Fonte: MS/SVS/CGIAE - Sistema de Informações sobre Mortalidade - SIM 
Quadro 2 - Óbitos por faixa etária, Piauí, 2010-2015

\begin{tabular}{|c|c|c|}
\hline Faixa Etária & $\begin{array}{c}\text { Óbitos por } \\
\text { Residência }\end{array}$ & $\%$ \\
\hline Menor 1 ano & 4711 & $4,5 \%$ \\
\hline 1 a 19 anos & 3740 & $3,5 \%$ \\
\hline 20 a 39 anos & 11830 & $11,2 \%$ \\
\hline 40 a 59 anos & 18595 & $17,5 \%$ \\
\hline$\geq 60$ anos & 67013 & $63,2 \%$ \\
\hline $\begin{array}{c}\text { Idade } \\
\text { ignorada }\end{array}$ & 142 & $0,1 \%$ \\
\hline Total & 10603 & $100 \%$ \\
\hline
\end{tabular}

Fonte: MS/SVS/CGIAE - Sistema de Informações sobre Mortalidade - SIM

Quadro 3 - Óbitos de menores de 1 ano segundo ano e faixa etária, Piaú, 2010- 2015

\begin{tabular}{|c|c|c|c|c|}
\hline Ano do Óbito & $\mathbf{0}$ a $\mathbf{6}$ dias & $\mathbf{7}$ a 27 dias & $\mathbf{2 8}$ a 364 dias & Total \\
\hline 2010 & $474(56,9 \%)$ & $132(15,9 \%)$ & $227(27,3 \%)$ & 833 \\
\hline 2011 & $484(57,1 \%)$ & $142(16,8 \%)$ & $221(26,1 \%)$ & 847 \\
\hline 2012 & $460(57,7 \%)$ & $129(16,2 \%)$ & $208(26,1 \%)$ & 797 \\
\hline 2013 & $436(57,3 \%)$ & $117(15,4 \%)$ & $208(27,3 \%)$ & 761 \\
\hline 2014 & $415(55,9 \%)$ & $130(17,5 \%)$ & $198(26,6 \%)$ & 743 \\
\hline 2015 & $413(56,6 \%)$ & $119(16,3 \%)$ & $198(27,1 \%)$ & 730 \\
\hline Total & 2682 & 769 & 1260 & $4711(\mathrm{n})$ \\
\hline
\end{tabular}

Fonte: MS/SVS/CGIAE - Sistema de Informações sobre Mortalidade - SIM

Gráfico 1 - Óbitos em menores de 1 ano de idade, segundo os anos, Piauí, 2010 a 2015

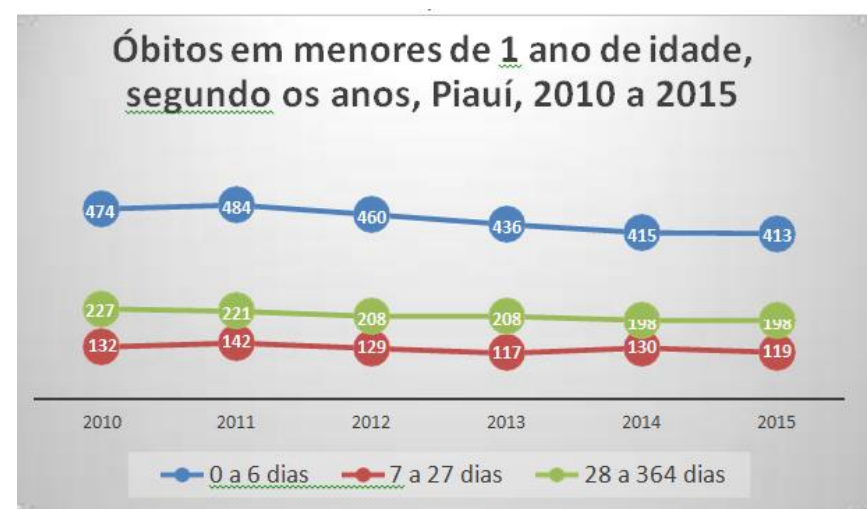

Fonte: MS/SVS/CGIAE - Sistema de Informações sobre

Mortalidade - SIM 
Quadro 4 - Óbitos segundo a idade e o sexo, Piauí, 2010 a 2015. N=106031

\begin{tabular}{|c|c|c|c|c|c|}
\hline \multirow{2}{*}{ IDADE } & \multicolumn{5}{|c|}{ SEXO } \\
\cline { 3 - 6 } & Masc & Fem & Ign & TOTAL & $\begin{array}{c}\text { Razão entre } \\
\text { os sexos }\end{array}$ \\
\hline Menor 1 ano & 2571 & 2074 & 66 & 4711 & 1,24 \\
\hline 1 a 19 anos & 2449 & 1291 & - & 3740 & 1,89 \\
\hline 20 a 29 anos & 4465 & 1148 & - & 5613 & 3,89 \\
\hline 30 a 39 anos & 4615 & 1600 & 2 & 6217 & 2,88 \\
\hline 40 a 49 anos & 5168 & 2592 & - & 7760 & 1,99 \\
\hline 50 a 59 anos & 6687 & 4147 & 1 & 10835 & 1,61 \\
\hline 60 a 69 anos & 8900 & 6240 & - & 15140 & 1,43 \\
\hline 70 a 79 anos & 11087 & 9527 & 1 & 20615 & 1,16 \\
\hline 80 anos e mais & 14788 & 16467 & 3 & 31258 & 0,90 \\
\hline Idade ignorada & 114 & 17 & 11 & 142 & 6,71 \\
\hline TOTAL & 60844 & 45103 & 84 & 106031 & 1,35 \\
\hline
\end{tabular}

Fonte: MS/SVS/CGIAE - Sistema de Informações sobre Mortalidade - SIM

Gráfico 2 - Percentual de óbitos segundo o sexo,

Piauí, 2010 a 2015. $\mathrm{N}=106031$

Percentual de óbitos segundo o sexo, Piauí, 2010 a 2015

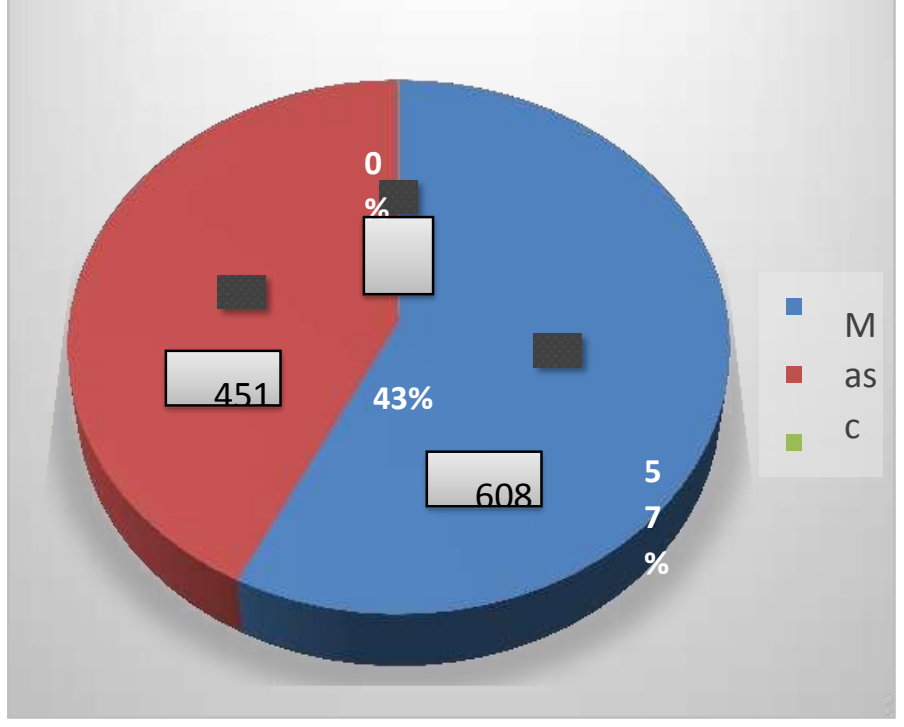

Fonte: MS/SVS/CGIAE - Sistema de Informações sobre Mortalidade - SIM 
Quadro 5 - Número e percentual de óbitos, segundo o capítulo do CID-10, Piauí, 2010 a 2015.

$\mathrm{N}=106031$

\begin{tabular}{|c|c|c|}
\hline Capítulo CID-10 & \multicolumn{2}{|c|}{ (n) Óbitos (\%) } \\
\hline I. Algumas doenças infecciosas e parasitárias & 3849 & $3,63 \%$ \\
\hline II. Neoplasias (tumores) & 14186 & $13,38 \%$ \\
\hline $\begin{array}{l}\text { III. Doenças do sangue e dos órgãos hematopoéticos e alguns } \\
\text { transtornos imunitários }\end{array}$ & 605 & $0,57 \%$ \\
\hline IV. Doenças endócrinas nutricionais e metabólicas & 8364 & $7,89 \%$ \\
\hline V. Transtornos mentais e comportamentais & 1239 & $1,17 \%$ \\
\hline VI. Doenças do sistema nervoso & 1972 & $1,86 \%$ \\
\hline VII. Doenças do olho e anexos & 3 & $0,00 \%$ \\
\hline VIII. Doenças do ouvido e da apófise mastóide & 14 & $0,01 \%$ \\
\hline IX. Doenças do aparelho circulatório & 36272 & $34,21 \%$ \\
\hline X. Doenças do aparelho respiratório & 8694 & $8,20 \%$ \\
\hline XI. Doenças do aparelho digestivo & 5203 & $4,91 \%$ \\
\hline XII. Doenças da pele e do tecido subcutâneo & 281 & $0,27 \%$ \\
\hline XIII. Doenças do sistema osteomuscular e do tecido conjuntivo & 400 & $0,38 \%$ \\
\hline XIV. Doenças do aparelho geniturinário & 1799 & $1,70 \%$ \\
\hline XV. Gravidez parto e puerpério & 298 & $0,28 \%$ \\
\hline XVI. Algumas afecções originadas no período perinatal & 3049 & $2,88 \%$ \\
\hline $\begin{array}{l}\text { XVII. Malformações congênitas, deformidades e } \\
\text { anomalias cromossômicas }\end{array}$ & 1191 & $1,12 \%$ \\
\hline $\begin{array}{l}\text { XVIII. Sintomas, sinais e achados anormais de exames clínicos e de } \\
\text { laboratório, não classificados em outra parte }\end{array}$ & 4778 & $4,51 \%$ \\
\hline XX. Causas externas de morbidade e mortalidade & 13834 & $13,05 \%$ \\
\hline Total & 106031 & $100 \%$ \\
\hline
\end{tabular}

Fonte: MS/SVS/CGIAE - Sistema de Informações sobre Mortalidade - SIM

\section{DSICUSÃO}

O presente estudo analisou os óbitos ocorridos no estado do Piauí, no período de 2010 a 2015, perfazendo uma amostra (n) no total de 106.031 óbitos. De acordo com o Quadro 1, que mostra os óbitos ocorridos no
Estado em cada ano do período em estudo, houve um aumento progressivo no número de óbitos no decorrer dos anos. O menor número de óbitos ocorreu em 2010, num total de 15.614 ( $14,7 \%$ do total) e o maior número ocorreu em 2015, correspondendo a $18,3 \%$ das mortes no período. Percebe-se, portanto, que, de acordo com esse quadro, de 2010 a 2015, ocorreu um aumento de 
3,5\% no número de óbitos notificados no Piauí. Pode-se observar que houve uma tendência crescente no número de óbitos no período.

De acordo com dados do Sistema de Informações sobre Mortalidade (SIM), tal aumento progressivo se deu também a nível nacional. Em 2010, ocorreram 1.136.947 óbitos, enquanto que em 2015 ocorreram $1.264 .175,1,8 \%$ a mais que no primeiro ano em análise.

Tal aumento mostrado no Quadro 1 pode ter como base as causas violentas, cujas estimativas apontam que, por ano, mais de um milhão das mortes no mundo sejam devido a elas ${ }^{(3)}$.

As doenças cardiovasculares, principal causa de óbitos no país, também são responsáveis por esse incremento, devido ao aumento do sedentarismo e da alimentação inadequada, rica em gorduras saturadas, que levaram ao aumento de dislipidemia e à hipertensão na população(7).

No período estudado, no estado do Piauí, os óbitos aumentaram percentualmente quase duas vezes quando comparados com os óbitos ao nível nacional. As causas para esses óbitos precisam ser melhor explicados em estudos posteriores.

Em relação à faixa etária dos óbitos ocorridos no período, observou-se, de acordo com o Quadro 2, que $4,5 \%$ de todas as mortes ocorridas entre 2010 e 2015 foram de crianças menores de 1 ano, 3,5\% na faixa etária de 1 a 19 anos, 11 ,2\% de adultos jovens ( 20 a 39 anos), $17,5 \%$ de adultos de meia idade (40 a 59 anos) e $63,2 \%$ dos óbitos foram de idosos (igual ou acima de 60 anos).

Nota-se a predominância da incidência de óbitos em idosos. No decorrer dos anos, ocorreram mudanças na pirâmide etária da população brasileira, com o aumento da população idosa, em consequência da redução das taxas de fecundidade e do aumento da expectativa de vida $^{(8)}$.

Em decorrência de tal aumento, ocorreu também elevação dos óbitos devido a doenças crônicas não transmissíveis (DCNT). A Organização Mundial da Saúde (OMS), em 2008, estimou que 38 milhões do total de óbitos a nível mundial (63\%) foram devido a DCNT, principalmente doenças do aparelho cardiovascular, diabetes, câncer e doenças pulmonares crônicas. No mesmo ano, estimou-se que apenas $29 \%$ dos óbitos por DCNT foram em menores de 60 anos, em países em desenvolvimento, enquanto que nos países desenvolvidos tal incidência foi de $13 \%{ }^{(2)}$.

Por tratar-se de um grupo bastante heterogêneo, cada faixa etária apresenta uma vulnerabilidade diferente em relação às distintas etiologias de óbitos. Adultos jovens e de meia idade são as principais vítimas de causas externas, dentre elas os acidentes de trânsito e as causas violentas, principalmente o homicídio. Estimase que o número de óbitos por causas violentas seja de mais de um milhão por ano no mundo, com a maior parcela desses óbitos ocorrendo em indivíduos na faixa de 15 a 44 anos de vida(3).

Nota-se, de acordo com o Quadro 3, discreta redução na mortalidade de menores de um ano, no decorrer dos anos em questão, visto que, em 2010, os óbitos nessa faixa etária corresponderam a 5,3\% dos óbitos gerais, caindo para 3,8\% em 2015. Tal redução corrobora com o que ocorreu em âmbito nacional. Em 2015, de acordo com dados de Estatísticas do Registro Civil, fornecidas pelo Instituto Brasileiro de Geografia e Estatística (IBGE), 2,5\% do total de óbitos no Brasil ocorreu nessa faixa etária, 1,5\% a menos do que em 2005.

No primeiro ano de vida, o risco de óbito está diretamente relacionado às condições socioeconômicas, como saneamento básico, nutrição, segurança alimentar, escolaridade materna e vacinação. Por essa razão, apesar da redução do número de óbitos nessa faixa etária, esse índice ainda é expressivo, visto que a maioria das causas de óbitos é evitável através de políticas públicas de atenção à saúde da criança $^{(9)}$.

Ainda de acordo com o quadro 3 , constata-se que a faixa etária de maior incidência de óbitos em menores de um ano é a dos neonatos, principalmente os neonatos precoces (0 a 6 dias de vida). Isso corrobora com a literatura, visto que, a mortalidade no período neonatal representa mais de $70 \%$ da mortalidade infantil no Brasil, com $25 \%$ desses óbitos nas primeiras 24 horas de vida, que integra o período neonatal precoce ${ }^{(10)}$. 
Isto posto, constata-se que, embora a mortalidade infantil esteja em queda, os óbitos de neonatos continuam elevados. Esse elevado índice de mortalidade neonatal está diretamente relacionado à assistência prestada à gestante durante o período prénatal, além da assistência ao parto e ao recémnascido ${ }^{(11)}$.

O Gráfico 1 mostra a distribuição dos 4711 óbitos que acometeram menores de 1 ano no período de 2010 a 2015 no estado do Piauí onde pode-se constatar que as maiores taxas de mortalidade aconteceram em recémnascidos menores de 7 dias, com 2682 óbitos (56,93\%), seguido da faixa etária de 28 a 364 dias, 1260 óbitos $(26,74 \%)$ e 769 mortes $(16,33 \%)$ de crianças entre 7 e 27 dias no decorrer do período estudado.

Em um estudo realizado em Cuiabá (MT), também percebeu-se a faixa neonatal como a mais prevalente entre os menores de 1 ano, principalmente no período neonatal precoce (de 0 a 6 dias de vida) ${ }^{(12)}$.

Ainda é possível observar no gráfico 1 que o ano de 2011 apresentou o maior número de casos dentre os anos estudados, 847 ( $18 \%$ dos óbitos), enquanto o ano de 2015 apresentou a menor quantidade de óbitos referente às variáveis estudadas, totalizando 730 óbitos $(15,5 \%)$. Durante o período não houve uma linearidade crescente ou decrescente, com alguns picos, mas podese avaliar que houve quedas nos números entre o primeiro ano de estudo (2010) e o último (2015).

Esses dados sugerem uma melhora no quadro da mortalidade infantil, haja visto que a diminuição nos números referentes à mortalidade infantil denota uma melhoria na saúde da mulher e da criança, principalmente no que se refere à qualidade dos serviços e assistência oferecida, além das condições socioeconômicas de uma determinada população ${ }^{(11)}$.

Estudos apontam que as políticas assistencialistas como - Bolsa Alimentação e Bolsa Família, associados à cobertura mais abrangente da atenção básica à gestante e ao recém-nascido, contribuíram positivamente na redução das taxas de mortalidade infantil, a partir da melhora dos indicadores sociais ${ }^{(12)}$.
Não foi possível correlacionar se os óbitos estão relacionados com a prematuridade, pré-natal ausente ou incompleto, baixo peso ao nascer entre outras comorbidades, pois os dados presentes no sistema não discriminavam a causa do óbito de acordo com esses fatores intrínsecos à mortalidade infantil.

A partir do Quadro 4 e do Gráfico 2, pode-se observar que o número de óbitos é maior entre os homens, 60844 mortes (57\%) do que em mulheres, 45103 óbitos (43\%), fato que não é proporcional à população brasileira em que as mulheres são maioria.

É preocupante observar que a razão entre os sexos na faixa etária é superior a 1,0 em quase todas as faixas etárias, com exceção dos idosos maiores que 80 anos, assim como apresentado no estudo de Souza e Siviero (2015). Observou-se que entre a faixa etária de 20 a 29 anos há uma grande disparidade entre os óbitos no sexo masculino e feminino chegando a razão de 3,89 , seguida de 15 a 19 anos $(3,04)$ e 30 a 39 anos $(2,88)$, tendo correspondência com outros estudos ${ }^{(13,14)}$.

De um modo geral, os homens jovens e adultos estão mais sujeitos à mortes por causas externas, principalmente nos acidentes de trânsito em que eles geralmente conduzem o veículo com maior velocidade, realizam manobras mais arriscadas do que as mulheres e ainda dirigem após a ingestão alcoólica ${ }^{(15)}$.

Estudos apontam que o fator biológico também influencia na diferença na mortalidade entre os sexos, desde 0 segundo cromossomo $X$ que impede as mulheres desenvolverem algumas doenças genéticas que se manifestam apenas em homens, além de maior adaptabilidade quanto às reservas energéticas correspondentes às necessidades da gestação e aleitamento. Ademais, também atribuem aos hormônios femininos (progesterona e estrogênio) como fator protetor de doenças circulatórias e cerebrovasculares $^{(14)}$.

O estilo de vida também contribui para esse hiato na mortalidade, visto que as mulheres de modo geral tem hábitos de vida mais saudável, com menor consumo de álcool, tabaco e drogas, procuram com mais frequência os serviços de saúde, praticam exercícios físicos, entre outras condições socioeconômicas ${ }^{(14,16)}$. 
Com base nos resultados apresentados no Quadro 5, verificou-se que a causa de óbito mais frequente foram as doenças do sistema circulatório (DSC) $(34,21 \%)$, seguida pelas neoplasias ou tumores $(13,38 \%)$, pelas causas externas $(13,05 \%)$ e doenças do sistema respiratório (8,20\%). A causa menos frequente foi por doenças do olho e seus anexos com apenas 3 registros notificados.

Os estudos de Alves e Monteiro (2016) e Oliveira, Medeiros e Lima (2015) também apresentam as doenças circulatórias como principal causa de óbitos, mas há divergências em relação à terceira etiologia mais frequente, visto que nessa pesquisa as causas externas ocupam essa posição, enquanto nas demais as doenças do aparelho respiratório, que aparecem logo após as doenças cardiovasculares e neoplasias ${ }^{(17,18)}$.

Um outro estudo também fez uma análise dos óbitos no Piauí no período entre 2005 e 2011 encontrando como principais causas: doenças relacionadas ao aparelho circulatório $(41,0 \%)$, neoplasias $(13,0 \%)$, causas externas de morbidade e mortalidade $(9,5 \%)$; doenças do aparelho respiratório (7,5\%)(19). Fazendo um paralelo entre os dados publicados por ele, houve uma redução dos óbitos por doenças circulatórias, no entanto, as neoplasias, doenças do aparelho respiratório e causas externas aumentaram no período de 2010 a 2015.

Essa redução nos óbitos por DSC pode ser justificada pela procura maior pelos serviços de saúde que diagnosticam mais precocemente essas comorbidades e consequentemente iniciam o tratamento em tempo hábil a evitar maiores complicações. Além disso, o acesso às orientações de estilo de vida mais saudável contribui com essa queda ${ }^{(18)}$.

Apesar da diminuição da mortalidade por doenças cardiovasculares, essas ainda constituem um importante problema de saúde pública que causam muitos danos ao organismo e à qualidade de vida, sendo necessário um rastreamento ativo e precoce dessas comorbidades, para oferecer uma melhor qualidade de vida para a população e proporcionar uma longevidade maior ${ }^{(7)}$.
$\mathrm{O}$ aumento dos registros de mortalidade por neoplasia deve-se ao aumento da exposição à fatores cancerígenos químicos, físicos e biológicos, pelo maior consumo de alimentos industrializados, pelo estilo de vida, pelo trabalho/estresse, entre outras causas que alteram a atividade celular(18).

Apesar do quadro 5 não discriminar qual a população mais acometida por óbitos de causas externas no Piauí, pesquisas revelaram que os adultos jovens (20 a 39 anos) do sexo masculino são os responsáveis por $25,5 \%$ das mortes nessa categoria, gerando grandes impactos emocional/familiar, financeiros, sociais e econômicos, que oneram o sistema de saúde e que vêm aumentando gradativamente, principalmente devido aos acidentes motociclísticos $(20,21)$.

\section{CONCLUSÃO}

O presente estudo permitiu caracterizar o perfil epidemiológico dos óbitos no estado do Piauí, entre 2010 e 2015, mostrando que houve um aumento progressivo no decorrer dos anos. O menor número de óbitos ocorreu em 2010, e o maior número ocorreu em 2015.

Em relação aos óbitos infantis, foi mensurado que a grande maioria está entre as crianças menores de um ano, cujos óbitos correspondem a mais da metade das mortes de crianças e adolescentes, e, entre esse grupo, especificamente, a faixa etária de maior incidência é a dos neonatos, principalmente os neonatos precoces $(0$ a 6 dias de vida).

Foi possível constatar que os pacientes do sexo masculino foram os que alcançaram a maior incidência em praticamente todas as faixas etárias, havendo predominância da incidência de óbitos em idosos, na faixa etária que compreende os indivíduos acima de 60 anos.

Entre as doenças que estavam na causa básica da morte foi possível classificar, em primeiro lugar as doenças do sistema circulatório, seguida pelas neoplasias ou tumores, pelos óbitos por causas externas e doenças do sistema respiratório. 0 estado do Piauí segue a 
tendência nacional e mundial de transição epidemiológica e demográfica, portanto, as políticas públicas voltadas para prevenção de mortes devem seguir essa mudança. Apesar da mortalidade infantil ter diminuído, ainda é necessário melhorar a assistência pré-natal, ao parto e ao recém-nascido.

Espera-se que os dados fornecidos do controle da mortalidade no Estado do Piauí sirvam de embasamento para a implantação de estudos em outros estados da federação, além de estratégias para novas políticas públicas, pois medir quantas pessoas morrem a cada ano e por que elas morreram é um dos meios mais importantes - juntamente com a avaliação de como as doenças e lesões estão afetando as pessoas - para avaliar a eficácia do sistema de saúde de um país.

\section{REFERÊNCIAS}

1. World Health Organization et al. Trends in maternal mortality: 1990 to 2010: WHO, UNICEF, UNFPA and The World Bank estimates. Trends in maternal mortality: 1990 to 2010: WHO, UNICEF, UNFPA and The World Bank estimates., 2017.

2. Malta DC et al. Mortalidade por doenças crônicas não transmissíveis no Brasil e suas regiões, 2000 a 2011. Epidemiologia e Serviços de Saúde, [internet] 2014;23:599-608. Disponível em: https://doi.org/10.5123/S1679-49742014000400002

\section{Mascarenhas MDM. Perfil epidemiológico dos} atendimentos de emergência por violência no Sistema de Serviços Sentinelas de Vigilância de Violências e Acidentes (Viva)-Brasil, 2006. Epidemiologia e Serviços de Saúde [internet] 2009;18(1): 17-28. Disponível em: http://dx.doi.org/10.5123/S1679-49742009000100003

4. Cerqueira D et al. Atlas da violência 2018. Rio de Janeiro: Instituto de Pesquisa Econômica Aplicada/ Fórum Brasileiro de Segurança Publica. 2018.

5. Ministério da Saúde (BR), Secretaria de Atenção à Saúde. Departamento de Ações Programáticas Estratégicas. Perfil da morbimortalidade masculina no Brasil [recurso eletrônico] / Ministério da Saúde,
Secretaria de Atenção à Saúde, Departamento de Ações Programáticas Estratégicas. - Brasília: Ministério da Saúde; 2018.

6. Oliveira CM, Bonfim CV, Guimarães MJB, Farias PG, Medeiros ZM. Mortalidade infantil: tendência temporal e contribuição da vigilância do óbito. Acta paul. enferm, [internet] 2016;29(3):282-90. Disponível em: https://doi.org/10.1590/1982-0194201600040 7. Ishitani LH et al. Desigualdade social e mortalidade precoce por doenças cardiovasculares no Brasil. Revista de Saúde Pública, [internet] 2006;40:684-91. Disponível em: https://doi.org/10.1590/S0034$\underline{89102006000500019}$

8. Freire GA et al. Mortalidade por causas externas em idosos no Paraná, Brasil de 2001-2010. Journal of Health Sciences, [internet] 2015;15(2). Disponível em: https://revista.pgsskroton.com/index.php/JHealthSci/a rticle/view/744

9. United Nations Children's Fund (UNICEF). Committing to child survival: a promise renewed. eSocialSciences; 2015.

10. Gaiva MAM, Fujimori E, Sato APS. Fatores de risco maternos e infantis associados à mortalidade neonatal. Texto \& Contexto-Enfermagem, Florianópolis, [internet] 2016;25(4). Disponível em: https://doi.org/10.1590/0104-07072016002290015

11. Rocha R et al. Mortalidade neonatal e evitabilidade: uma análise do perfil epidemiológico. Revista enfermagem. UERJ, Rio de Janeiro, [internet] 2011;19(1):114-20. Disponível em: https://pesquisa.bvsalud.org/portal/resource/pt/int$\underline{2276}$

12. Lima JC et al. Estudo de base populacional sobre mortalidade infantil. Revista Ciência \& Saúde Coletiva, [internet] 2017;22(3). Disponível em:

https://doi.org/10.1590/1413-81232017223.12742016

13. Souza MFM et al. Análise da mortalidade por homicídios no Brasil.

Epidemiologia e Serviços de Saúde, 2007;16(1):7-18. 
14. Siviero PCL, Turra CM, Rodrigues RN. Níveis e padrões do diferencial de mortalidade por sexo no município de SP, 1920 a 2005. Anais, p. 1- 21, 2010.

15. Cover DP, Nunes EAB, Carvalho KM. Mortalidade por Acidentes de Trânsito no Piauí: Caracterização dos Casos no Biênio 2012-2013. Rev. Uningá, [S.I.], [internet] 2016;49(1). Disponível em:

http://revista.uninga.br/index.php/uninga/article/view $\angle 1309$

16. Laurenti R, Jorge MHPM, Gotlieb SLD. Perfil epidemiológico da morbi-mortalidade masculina.Ciência \& Saúde Coletiva, [internet] 2005;10(1):35-46. Disponível em: https://doi.org/10.1590/S1413-81232005000100010

17. Alves MIC, Monteiro MFG. Diferenciais na estrutura de mortalidade dos idosos nas regiões metropolitanas brasileiras. Anais, p. 1-18, 2016.

18. Oliveira TC, Medeiros WR, Lima KC. Diferenciais de mortalidade por causas nas faixas etárias limítrofes de idosos. Rev. Bras. Geriatr. Gerontol. [internet] 2015;18(1):85-94. Disponível em: https://doi.org/10.1590/1809-9823.2015.14203
19. Lima IP, Mota ELA. Avaliação do impacto de uma intervenção para a melhoria da notificação da causa básica de óbitos no Estado do Piauí, Brasil. Epidemiol. Serv. Saúde, [internet] 2011;20(3):297-305. Disponível em: http://dx.doi.org/10.5123/S167949742011000300004

20. Silva MM, Meschial WC, Oliveira MLF. Mortalidade de adolescentes por causas externas no estado do Paraná: análise de dados oficiais. Revista Brasileira de Pesquisa em Saúde / Brazilian Journal of Health Research, [internet] 2011;18(3):17-23. Disponível em: https://doi.org/10.21722/rbps.v18i3.15738

21. Corassa RB et al. Evolução da mortalidade por causas externas em Diamantina (MG), 2001 a 2012. Cadernos Saúde Coletiva [internet] 2017;25(3). Disponível em: https://doi.org/10.1590/1414$\underline{462 \times 201700030258}$

Sources of funding: No

Conflict of interest: No

Accepted: 2019/02/06

Publishing: 2019/12/24

Corresponding Address: Eduardo Andrade Vasconcelos. Teresina, Piauí, Brasil. Email: eduandradev.96@gmail.com 